\title{
NDRG4 sensitizes CRC cells to 5-FU by upregulating DDIT3 expression
}

\author{
RUIKAI LI ${ }^{1,2^{*}}$, CHENXIANG HE $^{3 *}$, LIANGLIANG SHEN ${ }^{2,4}$, SHUAI WANG ${ }^{1,2}$, \\ YAO SHEN $^{2,4}$, FAN FENG ${ }^{1,2}$, JIAN ZHANG ${ }^{2,4}$ and JIANYONG ZHENG ${ }^{1,2}$ \\ ${ }^{1}$ Department of Gastrointestinal Surgery, Xijing Hospital; ${ }^{2}$ State Key Laboratory of Cancer Biology, \\ Fourth Military Medical University, Xi'an, Shaanxi 710032; ${ }^{3}$ Department of General Surgery, \\ Shanghai Fourth People's Hospital Affiliated to Tongji University of Medicine, Shanghai 200080; ${ }^{4}$ Department of \\ Biochemistry and Molecular Biology, Fourth Military Medical University, Xi'an, Shaanxi 710032, P.R. China
}

Received March 21, 2021; Accepted August 13, 2021

DOI: $10.3892 / \mathrm{ol} .2021 .13043$

\begin{abstract}
The incidence of colorectal cancer (CRC) has remained high in recent years, and 5-fluorouracil (5-FU) is a vital chemotherapeutic agent for its treatment. Our previous study reported that N-myc downstream-regulated gene 4 (NDRG4) plays a tumor-suppressive role in CRC, but the mechanisms associated with NDRG4 and 5-FU chemosensitivity remain unclear. The results of the present study demonstrate that NDRG4 sensitized CRC cells to 5-FU by upregulating DNA damage inducible transcript 3 (DDIT3). NDRG4 inhibited the proliferation of CRC cells and the activation of PI3K/AKT and ERK signaling. Furthermore, NDRG4 promoted CRC cell apoptosis induced by $5-\mathrm{FU}$. Mechanistic analyses revealed that NDRG4 upregulated DDIT3 expression, and that the proapoptotic effect of NDRG4 under 5-FU treatment conditions was dependent on DDIT3. These findings support the biological value of the association between NDRG4, DDIT3 and 5-FU chemosensitivity in CRC, and may advance the clinical treatment of CRC in the future.
\end{abstract}

Correspondence to: Professor Jian Zhang or Professor Jianyong Zheng, State Key Laboratory of Cancer Biology, Fourth Military Medical University, 169 Changle Road, Xincheng, Xi'an, Shaanxi 710032, P.R. China

E-mail: biozhangj@fmmu.edu.cn

E-mail: zhjy68@163.com

*Contributed equally

Abbreviations: CRC, colorectal cancer; DDIT3, DNA damage inducible transcript 3; ER, endoplasmic reticulum; 5-FU, 5-fluorouracil; NDRG4, N-myc downstream-regulated gene 4; PARP, poly-ADP-ribose polymerase

Key words: apoptosis, chemosensitivity, CRC, DDIT3, 5-FU, NDRG4

\section{Introduction}

Colorectal cancer (CRC) is the third most frequently occurring cancer type worldwide, and with a high mortality rate, accounted for $\sim 930,000$ deaths in 2020 (1). Surgery remains the principal CRC treatment method to achieve complete resection of the primary tumor and metastatic lesions (2). However, in a large number of cases, complete resection is difficult. As such, minimizing tumor size and inhibiting further growth and proliferation are the primary aims for patients whose tumors cannot be completely removed, or those who are unable to tolerate surgery, for which chemotherapy and radiotherapy are the key treatment options (3). In addition, adjuvant chemotherapy has been used to extend the lifespan of patients with CRC (4).

5-Fluorouracil (5-FU) is a thymidylate synthase inhibitor that prevents the methylation of deoxyuridine acid to deoxythymidine acid, and with notable anticancer properties, was one of the first therapeutic drugs developed for clinical cancer treatment $(5,6)$. Since 5 -FU is widely utilized as a first-line treatment, the incidence of CRC resistance to 5-FU is gradually increasing (5). Therefore, strategies for enhancing the chemosensitivity of CRC cells to 5-FU are urgently required.

$\mathrm{N}$-myc downstream-regulated gene 4 (NDRG4) belongs to the NDRG family, the members of which are expressed in a variety of human organs, and are associated with a wide range of biological processes, such as organ development, tumor inhibition, angiogenesis and growth regulation (7). NDRG4 plays a tumor-suppressive role in various cancer types, including pancreatic ductal and esophageal adenocarcinoma $(8,9)$. In addition, hypermethylation of the NDRG4 promoter is associated with gastric cancer tumorigenesis, and is a predictor of poor prognosis in patients with the disease (10). NDRG4 also plays an important role in CRC, as molecular analysis of the NDRG4 promoter region in stool samples can be used to screen for CRC (11).

The DNA damage inducible transcript 3 (DDIT3) gene encodes a member of the CCAAT/enhancer binding protein transcription factor family. DDIT3 is primarily involved in apoptosis associated with endoplasmic reticulum (ER) 
stress, as it enhances the biological function of the BH3-only protein BCL2 interacting mediator of cell death and inhibits the antiapoptotic function of BCL2 (12). In addition, DDIT3 can inhibit CRC by promoting the cell apoptosis (13). In terms of chemotherapeutic resistance, low expression levels of DDIT3 have been associated with the chemoresistance of lung cancer cells to cisplatin (14). The aim of the present study was to investigate the tumor-suppressive effect of NDRG4 in the SW480 and SW620 CRC cell lines, as well as whether NDRG4 enhanced the sensitivity of CRC cells to 5-FU, and the associated molecular mechanism. The results of the present study may indicate a novel mechanism that reflects the important role of NDRG4 in CRC inhibition.

\section{Materials and methods}

Cell lines. The SW480 and SW620 cell lines were purchased from Procell Life Science \& Technology Co., Ltd., (cat. no. CL-0223 and CL-0225, respectively) and cultured in Leibovitz's L15 medium (Thermo Fisher Scientific, Inc.) supplemented with $10 \%$ fetal bovine serum (FBS) (Gibco; Thermo Fisher Scientific, Inc.) at $37^{\circ} \mathrm{C}$ (100\% air). The $293 \mathrm{~T}$ cell line was purchased from Procell Life Science \& Technology Co., Ltd. (cat. no. CL-0005) and cultured in DMEM (Thermo Fisher Scientific, Inc.) supplemented with 10\% FBS (Gibco; Thermo Fisher Scientific, Inc.) at $37^{\circ} \mathrm{C}$ with $5 \% \mathrm{CO}_{2}$. All cell lines were authenticated by STR authentication.

Stable transfection. The NDRG4 lentivirus and its lentiviral vector GV358, were purchased from Shanghai GeneChem Co., Ltd. SW480 and SW620 cells were infected with lentivirus at a multiplicity of infection (MOI) of 10 and 80 for $24 \mathrm{~h}$, respectively. And the stably transfected cells were selected with $3 \mu \mathrm{g} / \mathrm{ml}$ puromycin (Beyotime Institute of Biotechnology) for 2 weeks. In subsequent experiments, puromycin was maintained at $0.5 \mu \mathrm{g} / \mathrm{ml}$. Short hairpin (sh)RNAs targeting the DDIT3 gene (shRNA-1, 5'-GATCCCTGCACC AAGCATGAACAATTCTCGAGAATTGTTCATGCTTGG TGCAGTTTTTG-3'; shRNA-2, 5'-GATCCTGAACGGCTC AAGCAGGAAATCTCGAGATTTCCTGCTTGAGCCGTT CATTTTTG-3'), and non-targeting shRNA-negative control (shRNA-NC 5'-GATCCCAACAAGATGAAGAGCACC AACTCGAGTTGGTGCTCTTCATCTTGTTGTTTTTG-3') were cloned into the lentiviral vector PLVshRNA-EGFP(2A) Puro by Inovogen Biotechnology Pvt. Ltd. According to the manufacturer's protocol, $4 \mu \mathrm{g}$ lentiviral shRNA plasmids were mixed with packaging vector (PAX2 plasmid) and envelope vector (PMD2G plasmid) at the mass ratio of $4: 3: 1$, and subsequently transfected into $293 \mathrm{~T}$ cells using $20 \mu \mathrm{l}$ Lipofectamine 2000 Transfection Reagent (Thermo Fisher Scientific, Inc.). Following incubation at $37^{\circ} \mathrm{C}$ with serum-free DMEM for $12 \mathrm{~h}$, the $293 \mathrm{~T}$ cells were changed to be cultured in complete DMEM at $37^{\circ} \mathrm{C}$ for $48 \mathrm{~h}$. The lentivirus particles were subsequently collected and purified from the cell supernatants through a $0.45 \mu \mathrm{m}$ filter, and meanwhile, the titer of the lentivirus was determined using the qPCR Lentivirus Titer kit (Applied Biological Materials, Inc.). Then SW480 cells in the logarithmic growth phase were added to the lentivirus suspension $(\mathrm{MOI}=10)$ and incubated at $37^{\circ} \mathrm{C}$ for $48 \mathrm{~h}$, after which the medium was discarded and the SW480 cells were cultivated with screening Leibovitz's L15 medium containing $3 \mu \mathrm{g} / \mathrm{ml}$ puromycin for 2 weeks to select the positive infected cells. In subsequent experiments, they were maintained with $0.5 \mu \mathrm{g} / \mathrm{ml}$ puromycin.

MTT assay. Cells were seeded into a 96-well plate with five biological replicates per group. Before detection, $20 \mu \mathrm{l}$ MTT solution (Beyotime Institute of Biotechnology) was added to each well. The medium was replaced with $150 \mu \mathrm{l}$ DMSO (Amresco, LLC) after $4 \mathrm{~h}$ at $37^{\circ} \mathrm{C}$, and the plates were then shaken for $10 \mathrm{~min}$. The OD values were determined at $570 \mathrm{~nm}$ using a microplate reader. To investigate the effect of NDRG4 on the proliferative ability of CRC cell lines, activity was detected at $0,1,2,3$ and 4 days of cell culture. To determine the effects of 5-FU on cell viability, different concentrations of 5 -FU $(5,10,20,40$ and $50 \mu \mathrm{g} / \mathrm{ml})$ were added to cells in the logarithmic growth phase, and the absorbance was measured $48 \mathrm{~h}$ after treatment.

Western blotting. Cells were lysed using RIPA lysis buffer (Beyotime Institute of Biotechnology), and the protein concentration was determined using a BCA protein assay kit (Thermo Fisher Scientific, Inc.). A total of $40 \mu \mathrm{g}$ protein per lane was separated by $10 \%$ or $15 \%$ SDS-PAGE and transferred onto PVDF membranes. The PVDF membrane was then blocked with TBST (Tween-20 at $0.1 \%$ ) containing $5 \%$ skim milk at room temperature for $1.5 \mathrm{~h}$. The following primary antibodies were diluted 1:1,000 in Primary Antibody Dilution Buffer (Beyotime Institute of Biotechnology): anti-NDRG4 (monoclonal, rabbit anti-human; cat. no. 9039; Cell Signaling Technology, Inc.), anti-DDIT3 (monoclonal, mouse anti-human; cat. no. 2895; Cell Signaling Technology, Inc.), anti-p-AKT (monoclonal, rabbit anti-human; cat. no. 4060; Cell Signaling Technology, Inc.), anti-AKT (monoclonal, rabbit anti-human; cat. no. 4685; Cell Signaling Technology, Inc.), anti-p-ERK (polyclonal, rabbit anti-human; cat. no. ab4819; Abcam), anti-ERK (polyclonal, rabbit anti-human; cat. no. ab17942; Abcam), anti-cleaved caspase-3 (polyclonal, rabbit anti-human; cat. no. 9661; Cell Signaling Technology, Inc.), anti-PARP (monoclonal, rabbit anti-human; cat. no. 9532; Cell Signaling Technology, Inc.), and anti- $\beta$-actin (monoclonal, mouse anti-human; cat. no. D191047; Sangon Biotech, Co., Ltd.). After incubated by the primary antibody overnight at $4^{\circ} \mathrm{C}$, the membrane was washed with TBST (Tween-20 at $0.1 \%$ ) at room temperature three times for $10 \mathrm{~min}$ each. Appropriate secondary antibodies derived from the same species as the primary antibodies (anti-rabbit IgG, HRP-linked; cat. no. 7074; Cell Signaling Technology, Inc.; and anti-mouse IgG, HRP-linked; cat. no. 7076; Cell Signaling Technology, Inc.) were diluted at 1:5,000 and added to the membranes, incubated at room temperature for $1 \mathrm{~h}$. After that, the membrane was washed with TBST three times for 10 min each, and then FDbio-dura ECL Kit (Hangzhou Fude Biological Technology Co., Ltd.) was added for visualization. The blots were detected using the Tanon 550 Imaging System (Tanon Science and Technology Co., Ltd.).

Reverse transcription-quantitative (RT-q) PCR and PCR array. The RNeasy Mini kit (Qiagen, Inc.) was used to extract total cellular RNA, from which cDNA was then synthesized using 
the PrimeScript RT-PCR kit (Takara Bio, Inc.) according to the manufacturers' protocols. qPCR was performed using qPCR SYBR-Green Master Mix (Shanghai Yeasen Biotechnology Co., Ltd.) per the manufacturer's protocol. The thermocycling conditions were as follows: Pre-denaturation at $95^{\circ} \mathrm{C}$ for $5 \mathrm{~min}$, then 40 cycles of denaturation at $95^{\circ} \mathrm{C}$ for $10 \mathrm{sec}$, annealing at $60^{\circ} \mathrm{C}$ for $20 \mathrm{sec}$ and extension at $72^{\circ} \mathrm{C}$ for $20 \mathrm{sec}$. Relative mRNA expression levels were determined using the $2^{-\Delta \Delta \mathrm{Cq}}$ method (15). The primer sequences were as follows: DDIT3 forward, 5'-GGAAACAGAGTGGTCATTCCC-3' and reverse, 5'-CTGCTTGAGCCGTTCATTCTC-3'; CASP7 forward, 5'-AGTGACAGGTATGGGCGTTC-3' and reverse, 5'-CGG CATTTGTATGGTCCTCTT-3'; and $\beta$-actin forward, 5'-CCT GGGCATGGAGTCCTGTG-3' and reverse, 5'-TCTTCATTG TGCTGGGTGCC-3'. For PCR array analysis, the extracted cDNA was used for with the real-time RT ${ }^{2}$ Profiler PCR Array (Qiagen, Inc.) according to the manufacturer's protocol.

TdT-UTP nick end labeling (TUNEL) assay. TUNEL assays were performed using the One Step TUNEL Apoptosis Assay Kit (Beyotime Institute of Biotechnology) according to the manufacturer's protocol. TUNEL regent was added to cells after $48 \mathrm{~h}$ of treatment with PBS or 5-FU. Each sample was observed by microscopy in five visual fields. Images of the cells were acquired using a fluorescence microscope.

Flow cytometry. Apoptosis assays were performed with cell lines using an Annexin V-FITC/PI apoptosis detection kit (Shanghai Yeasen Biotechnology Co., Ltd.) according to the manufacturer's protocol. The experiments were conducted $48 \mathrm{~h}$ after treatment with PBS or 5-FU. Flow cytometry was performed using a FC500 Flow Cytometer (Beckman Coulter Co., Ltd.), and the data were analyzed using FlowJo 10 software (FlowJo LLC).

Colony formation assay. Single cells ( 200 per dish) were seeded into cell culture dishes with a diameter of $6 \mathrm{~cm}$. After 18 days of culture at $37^{\circ} \mathrm{C}$, visible colonies ( $>50$ cells per colony) had formed and the culture was terminated. The medium was replaced every 3 days during the culture period. The colonies were then washed, fixed with $4 \%$ paraformaldehyde (Beyotime Institute of Biotechnology) at room temperature for $15 \mathrm{~min}$ and stained with crystal violet (Sigma-Aldrich; Merck KGaA) at room temperature for $20 \mathrm{~min}$. Finally, images of the stained colonies were captured, and the colonies were manually counted.

EdU staining. EdU staining was performed using the BeyoClick EdU Cell Proliferation Kit with Alexa Fluor 594 (cat. no. C0078S; Beyotime Institute of Biotechnology) according to the manufacturer's protocol. Cells in the logarithmic growth phase ( $24-48 \mathrm{~h}$ in culture) were used for detection. Images of the cells were captured using a fluorescence microscope.

Statistical analysis. All data are expressed as the mean \pm SD. Statistical analyses were performed using GraphPad Prism 8 (GraphPad Software, Inc.). Student's t-test was used to analyze two independent groups. For comparisons between multiple groups, ANOVA was applied; Sidak's multiple comparisons test was used following two-way ANOVA, and Dunnett's multiple comparisons test was used following one-way ANOVA. $\mathrm{P}<0.05$ was considered to indicate a statistically significant difference.

\section{Results}

NDRG4 inhibits the proliferation of CRC cells. SW480 and SW620 CRC cells overexpressing NDRG4 were successfully constructed through lentiviral infection. The NDRG4 protein levels of the overexpression cells were notably higher than those of their control counterparts (Fig. 1A). The MTT assay results indicated that the proliferative capacity of NDRG4-overexpressing cells was significantly lower than that of the control cells on days 1-4 of cell culture (Fig. 1B). The colony formation assay revealed that the NDRG4-overexpressing cells formed fewer visible clones than the control cells (Fig. 1C). Furthermore, EdU analysis indicted that the proliferation of NDRG4-overexpressing cells was inhibited relative to that of the control cells (Fig. 1D). These results indicate that NDRG4 inhibited CRC cell proliferation.

NDRG4 inhibits the activation of PI3K/AKT and ERK signaling. Since both the PI3K/AKT and ERK signaling pathways are associated with cellular proliferation (16), the levels of AKT and ERK phosphorylation can be measured to reflect their degrees of activation. Western blotting revealed decreased levels of p-AKT and p-ERK in NDRG4-overexpressing cells (Fig. 1E), indicating that NDRG4 inhibited the activation of the PI3K/AKT and ERK signaling pathways.

NDRG4 promotes 5-FU-induced CRC cell apoptosis. MTT assays were used to assess cell viability after $48 \mathrm{~h}$ of culture with different concentrations of 5-FU. The overexpression of NDRG4 significantly decreased the viability of cells treated with 5-FU at five different concentrations (Fig. 2A). Moreover, the survival rates of NDRG4-overexpressing SW480 and SW620 cells were decreased most significantly following treatment with 40 and $10 \mu \mathrm{g} / \mathrm{ml} 5$-FU, respectively. Therefore, subsequent experiments were carried out using these two concentrations of 5-FU. Flow cytometry was performed to detect apoptosis, which showed that NDRG4 overexpression increased the rates of SW480 and SW620 apoptosis induced by 5-FU, compared with those of the control cells; measurements were based on the percentage of annexin-V-positive cells (Q2 + Q3), and two-way ANOVA revealed that the interaction between NDRG4 and 5-FU was statistically significant (Fig. 2B). Subsequently, a TUNEL apoptosis assay was conducted, and the experimental results confirmed that NDRG4 overexpression increased the apoptotic rate induced by 5-FU, compared with that of control cells (Fig. 2C and D). Furthermore, expression of the apoptosis-associated molecules cleaved caspase-3 (C-caspase-3) and poly-ADP-ribose polymerase (PARP, the cleaved substrate of caspase) was also examined. The expression level of C-caspase-3 was increased in NDRG4-overexpressing cells compared with the control cells, indicating an increase in cellular apoptosis. After adding 5-FU, the expression of C-caspase-3 was notably increased, and the difference between NDRG4-overexpressing cells and control cells was 

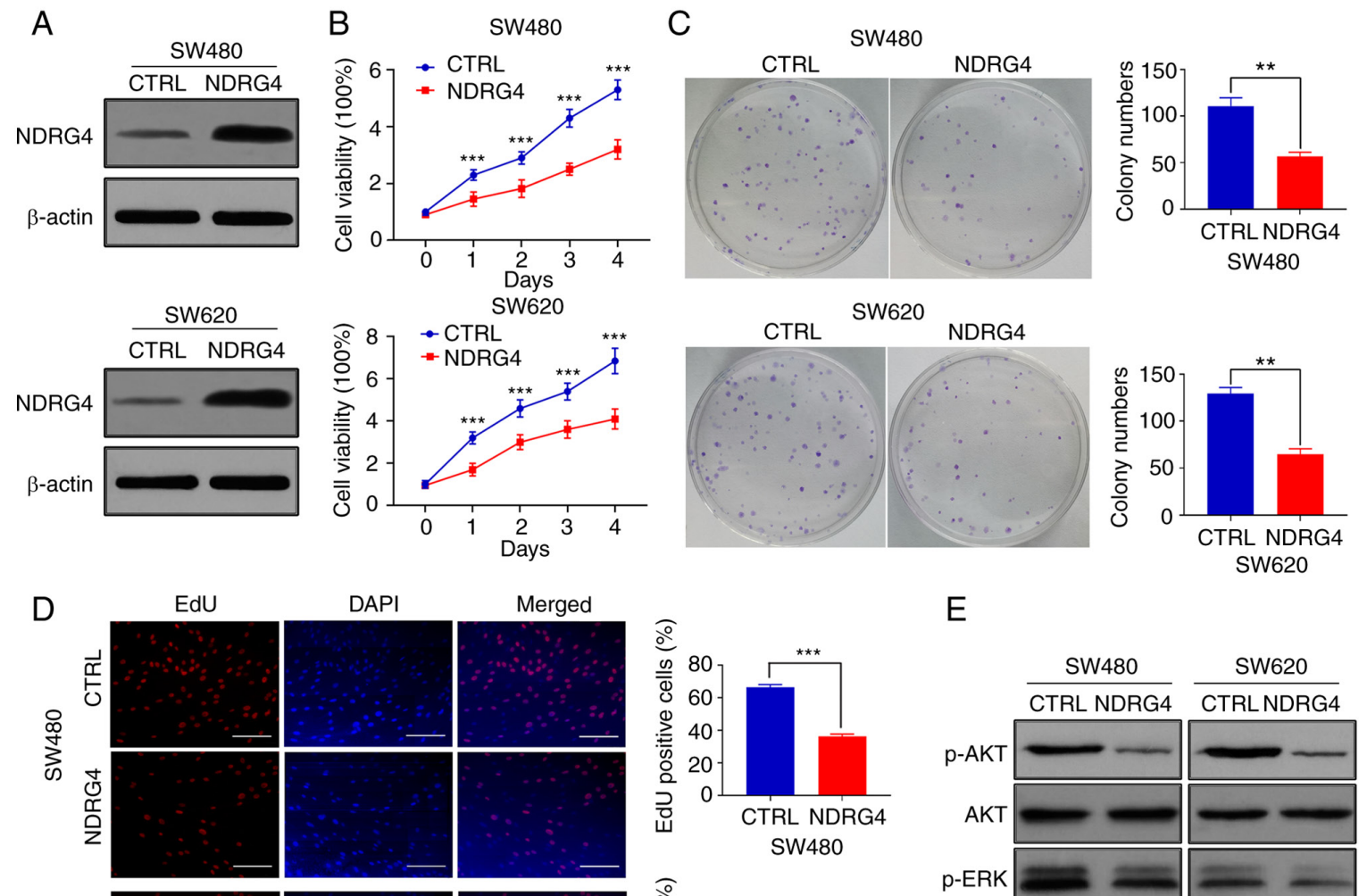

$\mathrm{E}$
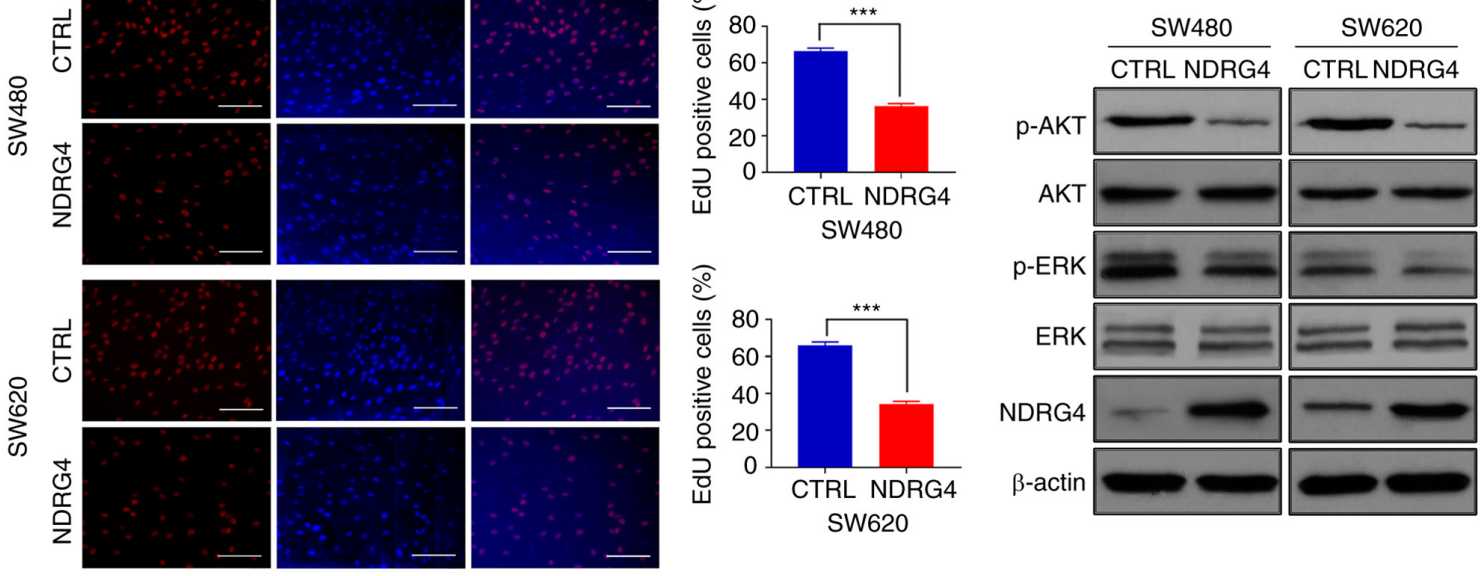

Figure 1. NDRG4 inhibits the proliferation of colorectal cancer cells. (A) Representative images of NDRG4 protein expression in SW480 and SW620 control and NDRG4-overexpressing cells detected by western blotting $(\mathrm{n}=3)$. (B) Viability of SW480 and SW620 control and NDRG4-overexpressing cells determined by MTT analysis. Statistical analysis by two-way ANOVA $(n=5)$. (C) Representative images and statistical analyses of colony formation capacity of control and NDRG4-overexpressing SW480 and SW620 cells. Statistical analysis by Student's t-test (n=3). (D) Representative images and the statistical analyses of SW480 and SW620 control and NDRG4-overexpressing cell proliferation, determined by the EdU analysis. Scale bar, $150 \mu \mathrm{m}$. Statistical analysis by Student's t-test $(n=3)$. (E) Representative images of p-AKT, AKT, p-ERK and ERK protein expression in SW480 and SW620 control and NDRG4-overexpressing cells detected by western blotting. Upper band of p-ERK represents phosphorylated ERK1, and the lower band represents phosphorylated ERK2. Upper band of ERK represents ERK1, and the lower band represents ERK2 (n=3). ${ }^{* * *} \mathrm{P}<0.001$ and ${ }^{* * *} \mathrm{P}<0.01$ vs. CTRL. NDRG4, N-myc downstream-regulated gene 4; p-, phosphorylated; CTRL, control.

still apparent. Meanwhile, NDRG4 overexpression and 5-FU treatment also increased PARP expression (Fig. 2E). These results suggest that NDRG4 promoted the apoptosis of CRC cells induced by 5 -FU.

NDRG4 upregulates DDIT3 expression. To investigate the molecular mechanism by which NDRG4 inhibits CRC cells, a PCR array experiment was conducted using SW480 cells to identify genes with considerable fold changes between NDRG4-overexpressing cells and control cells (Fig. 3A). Then, apoptosis-related genes, such as CASP7 and DDIT3, were selected for qPCR verification. DDIT3 exhibited the greatest differential expression between NDRG4-overexpressing cells and control cells (Fig. 3B). The western blot results also confirmed that DDIT3 was expressed at higher levels in NDRG4-overexpressing SW480 and SW620 cells than in their control counterparts (Fig. 3C). These results indicate that NDRG4 upregulated DDIT3 expression in CRC cells.
Proapoptotic effect of NDRG4 under 5-FU treatment is dependent on DDIT3. To further confirm whether the promotional effect of NDRG4 on 5-FU-induced CRC cell apoptosis was associated with the increase in DDIT3 expression, two shRNAs were designed to target the DDIT3 gene, and transfected into SW480 cells, which showed a successful decrease in DDIT3 mRNA expression (Fig. S1), and then into NDRG4-overexpressing SW480 cells to verify the gene silencing effect (Fig. 4A); further experiments were performed with shRNA-2, which exhibited the most prominent gene-silencing effect. The expression levels of the apoptosis-related proteins C-caspase-3 and PARP were decreased following DDIT3-knockdown in SW480 NDRG4-overexpressing cells. Silencing DDIT3 reduced the increase in C-caspase-3 and PARP expression induced by 5-FU treatment (Fig. 4B). Subsequently, SW480 NDRG4-overexpressing cells were analyzed by flow cytometry, revealing that DDIT3-knockdown resulted in a decreased apoptotic rate compared with that of control cells, and that the 
A
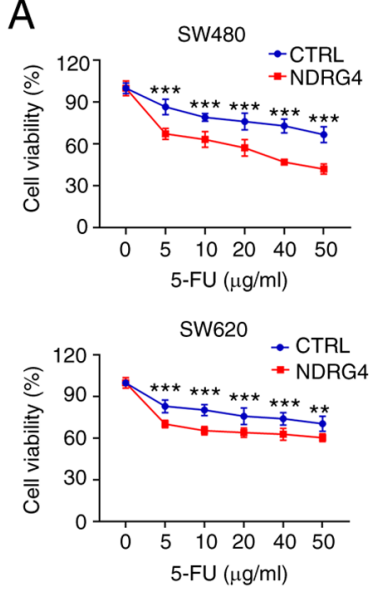

C

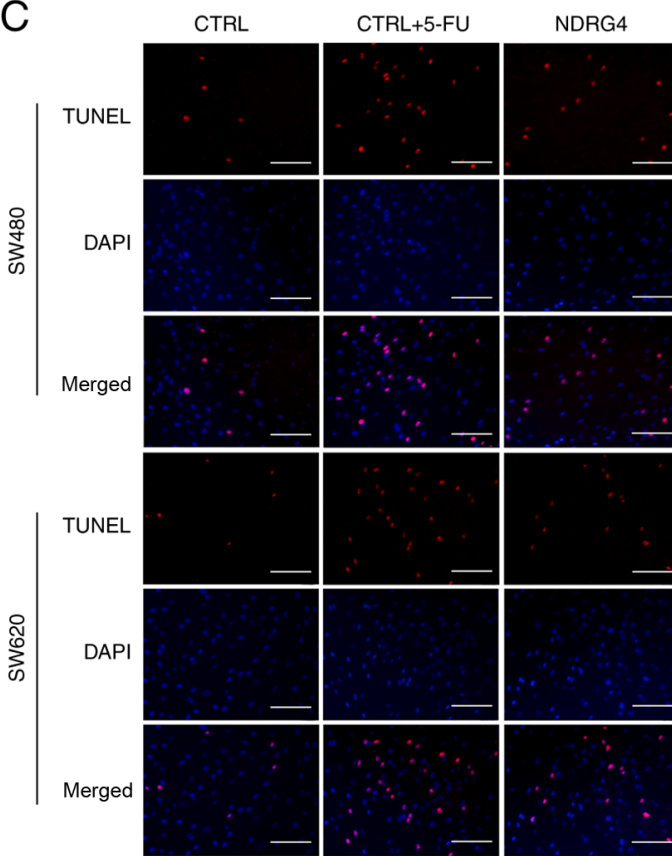

$\mathrm{B}$
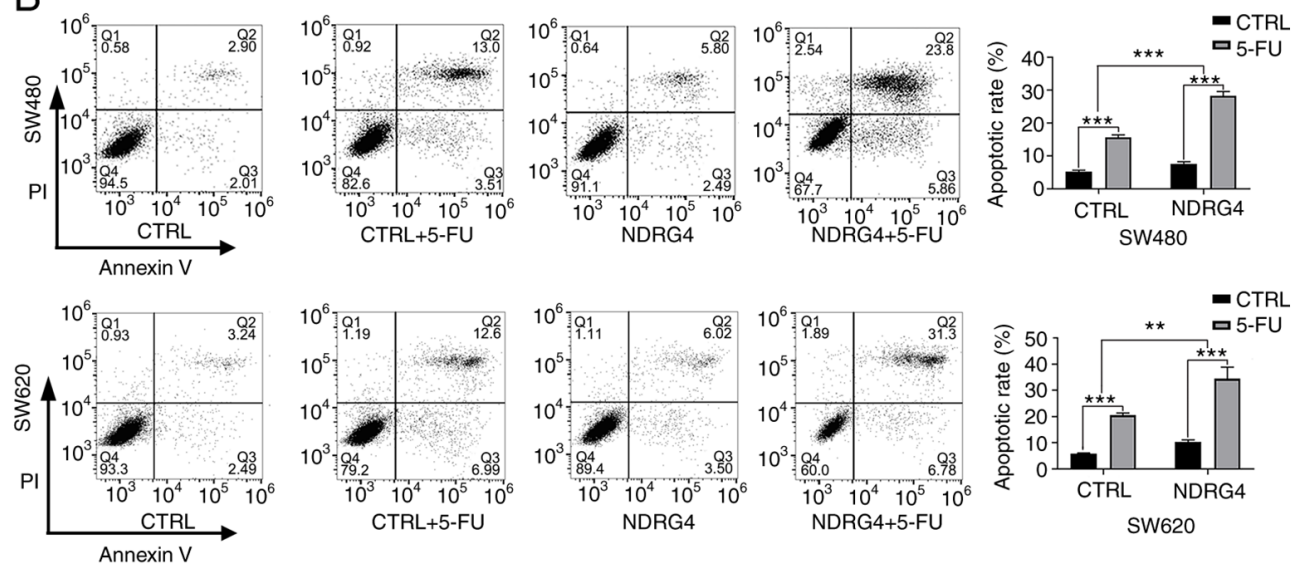
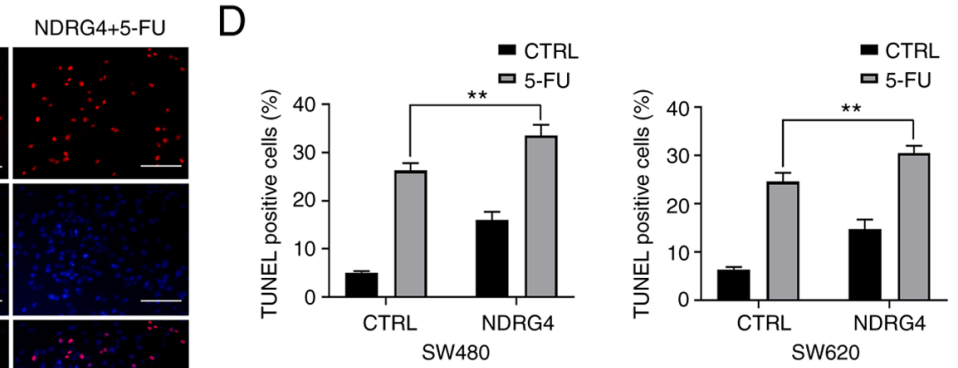

E

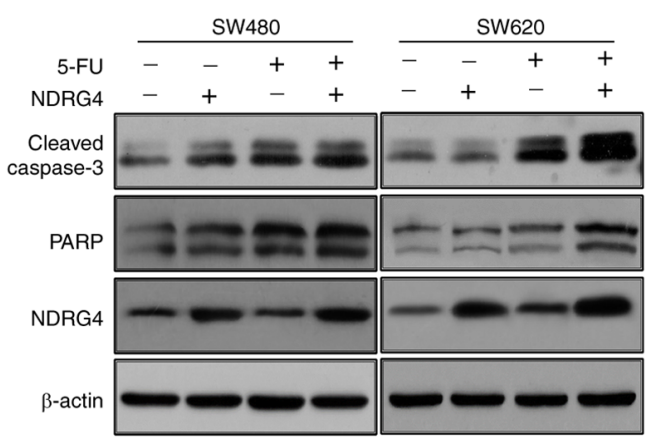

Figure 2. NDRG4 promotes 5-FU-induced colorectal cancer cell apoptosis. (A) Viability of SW480 and SW620 cells after $48 \mathrm{~h}$ of culture with different concentrations of 5-FU, determined by MTT assay. Statistical analysis by two-way ANOVA ( $\mathrm{n}=5$ ). (B) Detection of apoptosis in SW480 and SW620 control and NDRG4-overexpressing cells with or without 5-FU by flow cytometry. Top: Representative flow cytometric images and statistical analysis of the apoptotic rate of each group of SW480 cells. Bottom: Representative flow cytometric images and statistical analysis of the apoptotic rate of each SW620 cell group. Statistical analysis by two-way ANOVA (n=3). (C) Detection of apoptosis in SW480 and SW620 control and NDRG4-overexpressing cells with or without 5-FU by TUNEL assay $(\mathrm{n}=3)$. Scale bar, $150 \mu \mathrm{m}$. (D) Statistical analyses of the TUNEL assay results by two-way ANOVA. (E) Apoptosis-associated protein expression in SW480 and SW620 control and NDRG4-overexpressing cells with or without 5-FU as determined by western blotting. Multiple bands of C-caspase3 represent the large fragment $(17 / 19 \mathrm{kDa})$ of activated caspase-3 resulting from cleavage adjacent to Asp175. Upper band of PARP represents full-length PARP-1, and the lower band represents the large fragment produced by caspase cleavage at Asp214 ( $\mathrm{n}=3) .{ }^{* * * *} \mathrm{P}<0.001$ and ${ }^{* *} \mathrm{P}<0.01$ vs. CTRL. NDRG4, N-myc downstream-regulated gene 4; 5-FU, 5-fluorouracil; C-caspase, cleaved caspase; CTRL, control; PARP, poly-ADP-ribose polymerase; C-, cleaved.

decreasing trend was more apparent after the addition of 5-FU (Fig. 4C and D). These results indicate that DDIT3 plays an important role in the NDRG4-mediated promotion of CRC cell apoptosis induced by 5 -FU.

\section{Discussion}

The incidence and morbidity rates of CRC are high worldwide (1), and surgery remains the primary and most effective treatment type (17). In addition, adjuvant chemotherapy has been widely utilized to improve the survival of patients with both International Union Against Cancer (UICC) stage III CRC and high-risk UICC stage II CRC (18). Despite progress in cancer treatment, with the implementation of novel chemotherapeutic agents such as aflibercept, ramucirumab and bevacizumab, 5-FU remains one of the most effective and commonly used therapeutic drugs for CRC (19). Chemoresistance to anticancer agents is a major obstacle to attaining anticancer therapies with sufficient benefits (5), and the resistance of CRC to 5-FU is becoming increasingly prevalent.

Chemoresistance to 5-FU may be due to the disruption of 5-FU metabolic enzymes, drug transporters or crucial cellular activities, such as apoptosis and the cell cycle (20). For instance, Uppada et al (21) revealed that MASTL induces chemoresistance in colon cancer by promoting Wnt//-catenin signaling. Correspondingly, 5-FU sensitivity is influenced 
A

\begin{tabular}{ccc}
\hline Position & Gene & Fold regulation \\
\hline B03 & CASP7 & 2.21 \\
B05 & CCL2 & 2.05 \\
B12 & CPT2 & 2.12 \\
C02 & DDIT3 & 2.76 \\
C05 & E2F4 & 2.10 \\
D02 & G6PD & 3.26 \\
D03 & GADD45G & 2.85 \\
D05 & GSC & 2.22 \\
D10 & KDR & -2.16 \\
E02 & LPL & -2.22 \\
E07 & MKI67 & -2.35 \\
F07 & SNAl1 & 3.21 \\
F09 & SNAl3 & -6.80 \\
G01 & TBX2 & 2.29 \\
G10 & VEGFC & -2.02 \\
\hline
\end{tabular}

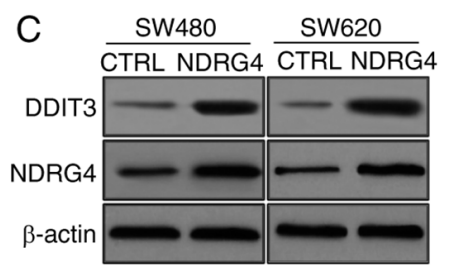

B
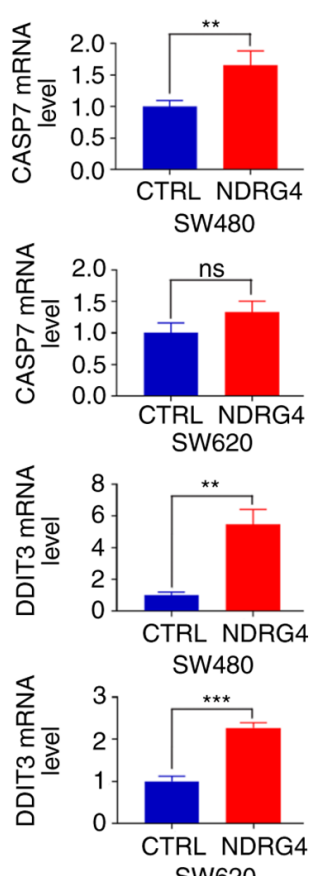

SW620

Figure 3. NDRG4 upregulates DDIT3 expression. (A) Genes with higher fold changes between SW480 NDRG4-overexpressing cells and control cells as determined by PCR array. (B) Reverse transcription-quantitative PCR verification of apoptosis-related gene expression in SW480 and SW620 control and NDRG4-overexpressing cells. Statistical analysis by Student's $\mathrm{t}$-test $(\mathrm{n}=3)$. (C) DDIT3 protein expression in SW480 and SW620 control and NDRG4-overexpressing cells as detected by western blotting $(\mathrm{n}=3){ }^{* * *} \mathrm{P}<0.001$ and ${ }^{* *} \mathrm{P}<0.01$. NDRG4, N-myc downstream-regulated gene 4; DDIT3, DNA damage inducible transcript 3; CTRL, control; ns, not significant.

by a variety of genes and chemical substances, and CDGSH iron-sulfur domain-containing protein 2 reportedly augments the chemosensitivity of gastric cancer by enhancing 5-FU-induced apoptosis (22). Another study indicated that dichloroacetate enhanced the chemosensitivity of CRC to 5-FU through vital metabolic pathways mediated by miRNAs (23).

The NDRG family contains four members, NDRG1-4, which share $57-65 \%$ identity at the amino acid level, and contain an $\alpha / \beta$ hydrolase-fold region (24). The four members have multiple biological functions (7). NDRG4 was reported to be expressed primarily in cells of the nervous system, including enteric neurons, suggesting its involvement in CRC through the enteric neuron system, and its potential as an early detection marker for CRC (25). However, the expression profile of NDRG4 has not been unified. Since human stool contains exfoliated intestinal epithelial cells (26), detecting the abnormal expression of certain molecules in stool (including NDRG4) has been used to screen for CRC, suggesting that NDRG4 may not be exclusively expressed in the enteric neurons in the colorectum. Although NDRG4 plays a tumor-suppressive role in various cancer types, the mechanism is rarely studied. Our previous study identified NDRG4 as a prognostic predictor for patients with CRC, and as a novel candidate tumor suppressor $(27,28)$. The present study revealed that NDRG4 inhibited the proliferation of SW480 and SW620 cells, which further confirmed the tumor-suppressive effect of NDRG4 in CRC. Interestingly, the effect of NDRG4 on chemosensitivity to 5-FU has not been previously reported.

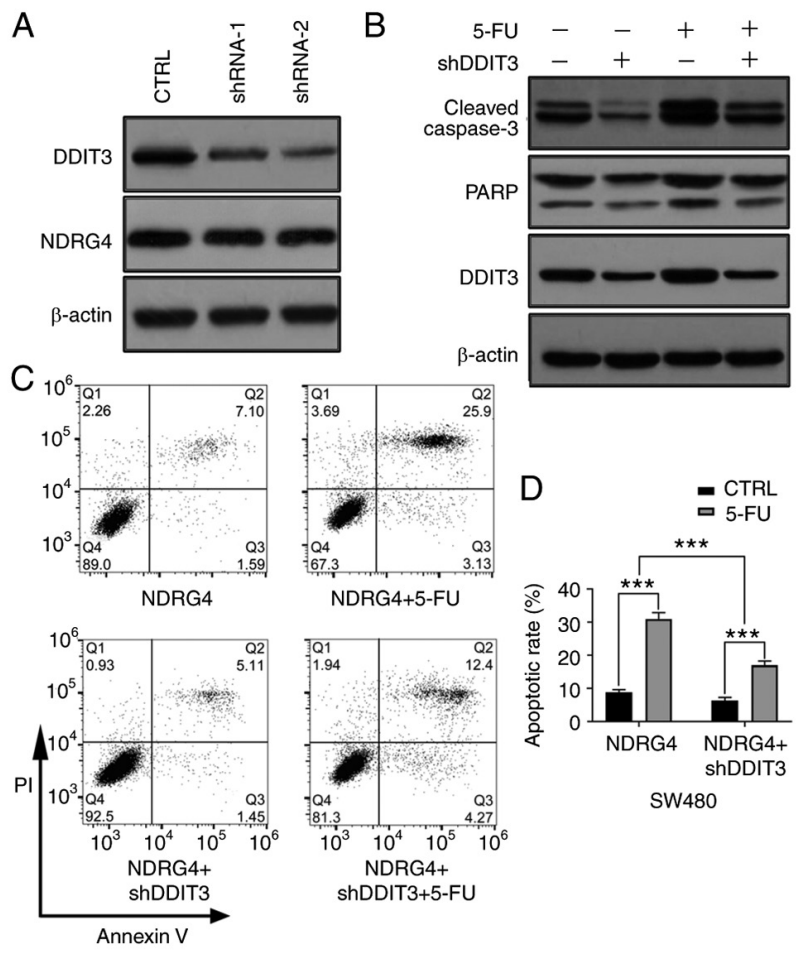

Figure 4. Proapoptotic effect of NDRG4 under 5-FU treatment is dependent on DDIT3. (A) Western blot analysis of NDRG4 and DDIT3 in SW480 NDRG4-overexpressing cells treated with or without DDIT3 shRNA $(n=3)$. (B) Apoptosis-associated protein expression in SW480 NDRG4-overexpressing cells with or without DDIT3-knockdown, and with or without 5-FU as determined by western blotting. Multiple C-caspase3 bands represent the large fragment $(17 / 19 \mathrm{kDa})$ of activated caspase-3 resulting from cleavage adjacent to Asp175. The upper band of PARP represents full-length PARP-1, and the lower band represents the large fragment produced by caspase cleavage at Asp214 (n=3). (C) Representative flow cytometric images of SW480 NDRG4-overexpressing cells with or without DDIT3-knockdown and with or without 5-FU treatment $(n=3)$. (D) Statistical analysis of the apoptotic rate of SW480 NDRG4-overexpressing cells with or without DDIT3-knockdown, and with or without 5-FU. Statistical analysis by two-way ANOVA. ${ }^{* * *} \mathrm{P}<0.001$. NDRG4, N-myc downstream-regulated gene 4; 5-FU, 5-fluorouracil; DDIT3, DNA damage inducible transcript 3; C-caspase, cleaved caspase; CTRL, control; PARP, poly-ADP-ribose polymerase; C-, cleaved; sh, short hairpin (RNA).

In the present study, a series of experiments was conducted to determine whether NDRG4 enhanced the sensitivity of CRC cells to 5-FU. The inhibitory effect of 5-FU on CRC cells has been reported to be positively correlated with its concentration (29). In order to determine the concentration used in subsequent experiments, five different concentrations of 5-FU were initially evaluated for their effects on the viability of NDRG4-overexpressing cells and control cells. The optimal concentration of 5-FU in SW480 and SW620 cells was 40 and $10 \mu \mathrm{g} / \mathrm{ml}$, respectively. The expression of apoptosis-related proteins was significantly increased in NDRG4-overexpressing cells compared with non-overexpressing cells when treated with 5-FU. However, the levels of NDRG4 were similar in NDRG4-overexpressing cells in the presence and absence of 5-FU, though the expression of apoptosis-related proteins was significantly higher in 5-FU treated NDRG4-overexpressing cells compared with untreated NDRG4-overexpressing cells (Fig. 2E). This suggests that 5-FU treatment did not affect the expression of NDRG4, but increased the expression of apoptosis-related proteins. 
Lu et al (30) demonstrated that treatment with 5-FU significantly increased the expression levels of C-caspase-3 and PARP, which is consistent with the results of the present study. The reason for the increased expression of apoptotic proteins may be that 5-FU drives the expression of apoptosis pathway genes by inducing conformational changes in the chromatin regions containing binding motifs for activator protein-1 family transcription factors (31).

To the best of our knowledge, no other studies have explored the relationship between NDRG4 and DDIT3 in CRC. However, there are reports supporting the association between DDIT3 and chemosensitivity. For example, Tan et al (14) noted that increasing the expression of DDIT3 enhanced the sensitivity of lung cancer cells to cisplatin. Another study reported that decreased expression of DDIT3 was an important factor underlying the 5-FU resistance of rectal cancer resulting from the high expression of rhomboid domain containing 2 (32). These reports are consistent with the findings of the present study; apoptosis experiments showed that NDRG4 overexpression enhanced the 5-FU-induced apoptosis of CRC cells, which was significantly weakened by DDIT3-knockdown in NDRG4-overexpressing SW480 cells, indicating the importance of DDIT3 in the apoptosis pathway.

DDIT3 is principally involved in ER stress-related apoptosis (33), and NDRG4 promotes the expression of DDIT3, suggesting that ER stress is associated with the tumor-suppressive effect of NDRG4. In addition, Zhang et al (34) observed that low DDIT3 expression was associated with the poor prognosis of patients with advanced gastric cancer, for whom it was suggested as a potential prognostic marker. Furthermore, as our previous studies showed that NDRG4 was associated with the prognosis of CRC $(27,28)$, DDIT3 may also be a prognostic biomarker for CRC.

Since SW480 cells are a classic CRC cell line with proliferative, invasive, migratory and tumorigenic characteristics, they are a commonly used model for the study of CRC in vitro. SW480 cells were primarily used in the present study, with partial verification studies conducted using the SW620 cell line. Therefore, PCR-array and DDIT3-knockdown experiments were not performed in SW620 cells, which is a study limitation. Organoids can simulate various real-organ characteristics, and are important models for studying disease (35). Considering that the enteric nervous system may play an important role in the function of NDRG4 (25), the use of intestinal organoids co-cultured with enteric neurons may be a future research prospect, along with the relevant molecular biological experiments, so as to further investigate the upstream and downstream molecular pathways of NDRG4. Of note, cell function and animal experiments were also not performed, and additional experiments, such as transwell assays and subcutaneous tumor-bearing experiments in athymic mice and tumor-specific patient-derived xenograft (PDX) models, will be a future consideration to further elucidate how NDRG4/DDIT3 regulates CRC cell responses to 5-FU treatment in vivo and in vitro.

In conclusion, the present study revealed that NDRG4 increased the chemosensitivity of CRC cells to 5-FU by increasing the expression of DDIT3, though the underlying mechanisms require further study in the future.

\section{Acknowledgements}

Not applicable.

\section{Funding}

The present study was supported by the National Natural Science Foundation of China (grant nos. 81572816 and 82072655).

\section{Availability of data and materials}

The datasets used and/or analyzed during the current study are available from the corresponding authors upon reasonable request.

\section{Authors' contributions}

JYZ and JZ conceived and designed the study and revised the manuscript. RKL and CXH performed most of the experiments and analyzed the data. RKL drafted the initial manuscript. LLS, SW and YS performed the MTT and TUNEL assays and analyzed the data. FF designed part of the study, analyzed the data and revised the manuscript. JZ and CXH confirmed the authenticity of all the raw data. All authors have read and approved the final manuscript.

\section{Ethics approval and consent to participate}

Not applicable.

\section{Patient consent for publication}

Not applicable.

\section{Competing interests}

The authors declare that they have no competing interests.

\section{References}

1. Sung H, Ferlay J, Siegel RL, Laversanne M, Soerjomataram I, Jemal A and Bray F: Global cancer statistics 2020: GLOBOCAN estimates of incidence and mortality worldwide for 36 cancers in 185 countries. CA Cancer J Clin 71: 209-249, 2021.

2. Kuipers EJ, Grady WM, Lieberman D, Seufferlein T, Sung JJ, Boelens PG, van de Velde CJ and Watanabe T: Colorectal cancer. Nat Rev Dis Primers 1: 15065, 2015.

3. Xie YH, Chen YX and Fang JY: Comprehensive review of targeted therapy for colorectal cancer. Signal Transduct Target Ther 5: 22, 2020 .

4. Brown KGM, Solomon MJ, Mahon K and O'Shannassy S: Management of colorectal cancer. BMJ 366: 14561, 2019.

5. Vodenkova S, Buchler T, Cervena K, Veskrnova V, Vodicka P and Vymetalkova V: 5-fluorouracil and other fluoropyrimidines in colorectal cancer: Past, present and future. Pharmacol Ther 206: $107447,2020$.

6. Xie P, Mo JL, Liu JH, Li X, Tan LM, Zhang W, Zhou HH and Liu ZQ: Pharmacogenomics of 5-fluorouracil in colorectal cancer: Review and update. Cell Oncol (Dordr) 43: 989-1001, 2020.

7. Yu C, Hao X, Zhang S, Hu W, Li J, Sun J and Zheng M: Characterization of the prognostic values of the NDRG family in gastric cancer. Therap Adv Gastroenterol 12: 1756284819858507 , 2019.

8. Shi HH, Liu HE and Luo XJ: Hypermethylation-mediated silencing of NDRG4 promotes pancreatic ductal adenocarcinoma by regulating mitochondrial function. BMB Rep 53: 658-663, 2020. 
9. Cao L, Hu T, Lu H and Peng D: N-MYC downstream regulated gene 4 (NDRG4), a frequent downregulated gene through DNA hypermethylation, plays a tumor suppressive role in esophageal adenocarcinoma. Cancers (Basel) 12: 2573, 2020.

10. Chen X, Yang Y, Liu J, Li B, Xu Y, Li C, Xu Q, Liu G, Chen Y, Ying $\mathrm{J}$ and Duan S: NDRG4 hypermethylation is a potential biomarker for diagnosis and prognosis of gastric cancer in Chinese population. Oncotarget 8: 8105-8119, 2017.

11. Kadiyska T and Nossikoff A: Stool DNA methylation assays in colorectal cancer screening. World J Gastroenterol 21: 10057-10061, 2015.

12. Puthalakath H, O'Reilly LA, Gunn P, Lee L, Kelly PN, Huntington ND, Hughes PD, MichalakEM,McKimm-Breschkin J, Motoyama N, et al: ER stress triggers apoptosis by activating BH3-only protein Bim. Cell 129: 1337-1349, 2007.

13. Li L, Chen Q, Yu Y, Chen H, Lu M, Huang Y, Li P and Chang H: RKI-1447 suppresses colorectal carcinoma cell growth via disrupting cellular bioenergetics and mitochondrial dynamics. J Cell Physiol 235: 254-266, 2020.

14. Tan W, Liao Y, Qiu Y, Liu H, Tan D, Wu T, Tang M, Zhang S and Wang $\mathrm{H}$ : MiRNA 146a promotes chemotherapy resistance in lung cancer cells by targeting DNA damage inducible transcript 3 (CHOP). Cancer Lett 428: 55-68, 2018.

15. Livak KJ and Schmittgen TD: Analysis of relative gene expression data using real-time quantitative PCR and the 2(-Delta Delta $\mathrm{C}(\mathrm{T})$ ) method. Methods 25: 402-408, 2001.

16. Cao Z, Liao Q, Su M, Huang K, Jin J and Cao D: AKT and ERK dual inhibitors: The way forward? Cancer Lett 459: 30-40, 2019.

17. Dekker E, Tanis PJ, Vleugels JLA, Kasi PM and Wallace MB: Colorectal cancer. Lancet 394: 1467-1480, 2019.

18. Stintzing S: Management of colorectal cancer. F1000Prime Rep 6: 108, 2014.

19. Sargent D, Sobrero A, Grothey A,O'Connell MJ,Buyse M,Andre T, Zheng Y, Green E, Labianca R, O'Callaghan C, et al: Evidence for cure by adjuvant therapy in colon cancer: Observations based on individual patient data from 20,898 patients on 18 randomized trials. J Clin Oncol 27: 872-877, 2009

20. Blondy S, David V, Verdier M, Mathonnet M, Perraud A and Christou N: 5-Fluorouracil resistance mechanisms in colorectal cancer: From classical pathways to promising processes. Cancer Sci 111: 3142-3154, 2020

21. Uppada SB, Gowrikumar S, Ahmad R, Kumar B, Szeglin B Chen X, Smith JJ, Batra SK, Singh AB and Dhawan P: MASTL induces colon cancer progression and chemoresistance by promoting Wnt $/ \beta$-catenin signaling. Mol Cancer 17 : $111,2018$.

22. Sun Y, Jiang Y, Huang J, Chen H, Liao Y and Yang Z: CISD2 enhances the chemosensitivity of gastric cancer through the enhancement of 5-FU-induced apoptosis and the inhibition of autophagy by AKT/mTOR pathway. Cancer Med 6: 2331-2346, 2017.

23. Liang Y, Hou L, Li L, Li L, Zhu L, Wang Y, Huang X, Hou Y, Zhu D, Zou H, et al: Dichloroacetate restores colorectal cancer chemosensitivity through the p53/miR-149-3p/PDK2-mediated glucose metabolic pathway. Oncogene 39: 469-485, 2020
24. Qu X, Zhai Y, Wei H, Zhang C, Xing G, Yu Y and He F: Characterization and expression of three novel differentiation-related genes belong to the human NDRG gene family. Mol Cell Biochem 229: 35-44, 2002.

25. Vaes N, Lentjes MHFM, Gijbels MJ, Rademakers G, Daenen KL, Boesmans W, Wouters KAD, Geuzens A, Qu X, Steinbusch HPJ, et al: NDRG4, an early detection marker for colorectal cancer, is specifically expressed in enteric neurons. Neurogastroenterol Motil 29: 2017.

26. Kisiel JB, Klepp P, Allawi HT, Taylor WR, Giakoumopoulos M, Sander T, Yab TC, Moum BA, Lidgard GP, Brackmann S, et al: Analysis of DNA methylation at specific loci in stool samples detects colorectal cancer and high-grade dysplasia in patients with inflammatory bowel disease. Clin Gastroenterol Hepatol 17: 914-921 e5, 2019.

27. Chu D, Zhang Z, Zhou Y, Li Y, Zhu S, Zhang J, Zhao Q, Ji G, Wang W and Zheng J: NDRG4, a novel candidate tumor suppressor, is a predictor of overall survival of colorectal cancer patients. Oncotarget 6: 7584-7596, 2015

28. Zheng J, Li Y, Zhu S, Li J, Zhao Q, Ji G, Wang W and Chu D: NDRG4 stratifies the prognostic value of body mass index in colorectal cancer. Oncotarget 7: 1311-1322, 2016.

29. Milczarek M, Rossowska J, Klopotowska D, Stachowicz M, Kutner A and Wietrzyk J: Tacalcitol increases the sensitivity of colorectal cancer cells to 5 -fluorouracil by downregulating the thymidylate synthase. J Steroid Biochem Mol Biol 190: 139-151, 2019.

30. Lu P, Xu M, Xiong Z, Zhou F and Wang L: Fusobacterium nucleatum prevents apoptosis in colorectal cancer cells via the ANO1 pathway. Cancer Manag Res 11: 9057-9066, 2019.

31. Yang CM, Kang MK, Jung WJ, Joo JS, Kim YJ, Choi Y and Kim HP: p53 expression confers sensitivity to 5-fluorouracil via distinct chromatin accessibility dynamics in human colorectal cancer. Oncol Lett 21: 226, 2021.

32. Palma S, Raffa CI, Garcia-Fabiani MB, Ferretti VA, Zwenger A, Perez Verdera PV, Llontop A, Rojas Bilbao E, Cuartero V, Abba MC and Lacunza E: RHBDD2 overexpression promotes a chemoresistant and invasive phenotype to rectal cancer tumors via modulating UPR and focal adhesion genes. Biochim Biophys Acta Mol Basis Dis 1866: 165810, 2020.

33. Yao RQ, Ren C, Xia ZF and Yao YM: Organelle-specific autophagy in inflammatory diseases: A potential therapeutic target underlying the quality control of multiple organelles. Autophagy 17: 385-401, 2021.

34. Zhang X, Zhou T, Li W, Zhang $\mathrm{T}$, Che $\mathrm{N}$ and $\mathrm{Zu} \mathrm{G}$ : Clinicopathological and prognostic significance of C/EBP homologous protein $(\mathrm{CHOP})$ in advanced gastric cancer. Pathol Res Pract 214: 1105-1109, 2018.

35. Ji DB and Wu AW: Organoid in colorectal cancer: Progress and challenges. Chin Med J (Engl) 133: 1971-1977, 2020.

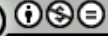

This work is licensed under a Creative Commons Attribution-NonCommercial-NoDerivatives 4.0 International (CC BY-NC-ND 4.0) License. 